\title{
Libraries, Archives, and Museums: Achieving Scale and Relevance in the Digital Age
}

OVER THE YEARS, I have heard many apologia for libraries, archives, and museums: why they are important, why they matter, and why we should care. In most cases, particularly in North America, the defense at some point invokes the role that cultural institutions play in the structure of a successful democracy: their support for the free flow of information that makes for an informed citizenry, and the preservation of a record of knowledge that enables choice and progress. Despite the fact that they are integrally bound up with American culture, I endorse all of those arguments; yet I do not feel they adequately explain the roles that cultural institutions play in society. I believe that the importance of cultural institutions derives from something much deeper, more universal, and more enduring.

I think that libraries, archives, and museums contribute to humankind's ability to become more robust and adaptable over the very long term. This is a vitally important role for global civilization, but one that can only be recognized from a higherlevel perspective. Cultural institutions - which many of us by now have become comfortable in calling "memory institutions"-are a crucial bedrock component in the overall order of civilization.

Consider civilization as a system. How has the system changed over time? How has it evolved? How does it absorb shock? How does it incorporate new information? In some respects, civilization is no different from any other adaptable system that depends on the relationships among its components for survival. But each of those individual components has its own scale and size, and differs in the rate at which it can change.

Much of my thinking about systems has been influenced by Stewart Brand, founder of the Long Now Foundation, and creator of the Whole Earth Catalogue. Brand's Clock of the Long Now: Time and Responsibility is a series of connected meditations and reflections on the meaning of time and civilization, and the ways in which 
we perceive ourselves. ${ }^{1}$ I have been impressed by Brand's ideas about the order of civilization, especially his theories concerning a healthy civilization and the layers of which it is composed. According to Brand, these layers support differing rates of change that allow the system to remain stable and to improve over time. I have found his way of thinking a useful heuristic for making sense of the kinds of work that cultural institutions are engaged in and the constituents they serve.

So how does human civilization absorb shocks and incorporate change? As an adaptable system, it does not simply break under stress. Instead, it yields. Some parts respond quickly to the shock, which in turn allows the slower parts to maintain the system's overall operation. This combination of fast and slow components makes the system as a whole resilient. Bland explains that the fast parts learn while the slow parts remember. These "fast parts" represent discontinuity, while the "slow ones" stand for continuity. The faster, smaller elements instruct the bigger, slower ones, which in turn serve to accrue innovation and permit us to remember it.

Brand claims that human civilization has a working structure composed of six levels of pace and size. The fastest level, or layer, consists of fashion and art. This is the layer where we experiment, where we move quickly and expect things to change rapidly. Beneath this layer is commerce, which feeds on fashion and art to create goods and services. Next is the infrastructure layer, which supports commerce and general progress. Supporting infrastructure is governance, and below that is culture, which ultimately gives governance its mandate. Finally, at the very bedrock level, is nature, the slowest and longest of the layers. In society, each of these layers operates at its own pace. The faster layers are sustained by the slower moving layers beneath them.

I believe that libraries, museums, and archives operate at this deep cultural layer of civilization, where memory institutions function as such. This is where we keep time in centuries, in millennia. It is much slower than political and economic history. Its pace is that of language and religion. It is the work of entire peoples. Memory institutions collect, maintain, and provide access to the cultural record. The contents of these institutions are what permit us to reinvent, to innovate, to grow, and to progress at all the other layers of civilization. They inform us about what we know. They help us understand how we govern. They dictate and describe the nature of our infrastructure. They provide the record of our commerce and even shape the fashion and art that we create.

1. Stewart Brand, The Clock of the Long Now: Time and Responsibility (New York: Basic Books, 2000). For more about the Long Now Foundation, see http:/ / www.longnow.org/. 
What makes libraries, archives, and museums so important is that they operate within this deep layer of culture and can therefore impact the other layers of civilization. Yet there is also an important corollary to this role: memory institutions have an enormous obligation to provide the broadest possible access to their contents, for that is what civilization needs from them.

If we cannot discover or gain access or interact with this slowly moving layer of culture, human progress is ultimately affected because the implications of that ignorance rebound throughout all the other layers. If part of our accumulated past is inaccessible, then we and future generations are denied resources for innovation and progress. Future generations will suffer because they will be forced to reinvent or rediscover what could have been learned from the past.

Each type of memory institution—libraries, archives, and museums - makes a unique contribution to our awareness and knowledge of the accumulated past. It may be useful to consider how that contribution is shaped by the various types of collections that are maintained by each of these institutional sectors. For instance, can the collections be equated in some way with sensory memory, short-term memory, and long-term memory? Perhaps this is not the best model, but it strikes me that the heuristic value in thinking of civilization as a complex of layers moving at different rates of speed holds much potential for thinking about the role of libraries, archives, and museums, both collectively and distinctly.

But along with the corollary of discoverability and access comes the converse of loss and forgetfulness. Think about the visceral reactions we have when we hear that a library has burned down, or an archives has been bombed, or a museum object has been stolen. The physical loss of cultural objects affects us. We bemoan such losses, as we should. Such a loss does not need to be explained. We just feel it.

Yet I submit that the real challenge we all now face is related to a different kind of a loss. It is not as much a question of physical loss or the need for physical preservation, but rather the challenges that have been brought about by the creation of digital information, digital discovery, and digital delivery. The whole digital information paradigm has effectively created a digital curtain in front of our physical collections. In many ways, our collections are lost behind that curtain, because they themselves are no longer visible within the new paradigm. We have a challenge and an obligation to raise the curtain. We have been talking about technology in libraries, archives, and museums for a long time now, but I genuinely think that as cultural institutions we are at a tipping point in terms of our relevance in the new information paradigm. Our users - the scholars, students, and citizens at-large for whom we maintain our collections-seem increasingly unaware of what we 
have and how they might use it because they are becoming accustomed to going elsewhere to discover what exists, even if these means provide only a surrogate for the "real" thing.

While I recognize that libraries, archives, and museums each have distinct characteristics and face different issues, I nevertheless think that our responses to the challenges of the digital age will share certain basic characteristics. Furthermore, I would suggest if our institutions-particularly those that have unique objects, unique documents, and unique published texts—are going to respond to their responsibilities and obligations in the new digital paradigm, we must recognize and accept two facts: 1) we cannot do it alone, and 2) we cannot do it using our current practices and cost structures.

We cannot do it alone because we cannot achieve visibility by ourselves. We have to collaborate. We have to create scale to create awareness. We have to ignore institutional competitiveness. And we have to acknowledge this fact as an imperative because the cultural record is scattered across many institutions, large and small.

Our world, to borrow the neologism of OCLC Chief Strategist Lorcan Dempsey, is the "Amazoogle" world. It is a world in which the information and discovery agenda is set without any significant involvement from our institutions. One of the reasons I led RLG into the combination with OCLC is because I believe that individual institutions will only be able to have an impact if we create mechanisms to aggregate what we do on a scale that is comparable to, and as important and responsive as, the commercial Web-scale players. Once that occurs, then we can think about how to bring our special knowledge of our users to bear on the creation of particular tools or perspectives or views that will inject our values and demonstrate our importance to the cultural progress of human civilization.

We must put our materials in front of people where they are doing their work. We need to ensure that our best collective memory is part of the global information flow that people around the world rely upon and now take for granted. We can be responsive players and participants in this new digital paradigm if we can move our knowledge in the aggregate up to the network level where it is visible on a global scale. I think that it is through such organizations as RLG, OCLC, and the others on which libraries, archives, and museums rely that we can create this sort of trusted collective presence and bring the knowledge held by our cultural institutions most effectively into the new digital environment.

We will not be able to achieve network levels of aggregation and scale, however, without dramatic changes in our practices. If we want to achieve global visibility, 
we are going to have to change the way we do business because the ways we do it now simply will not scale up to the level necessary to break through the digital curtain. We are not currently placing our collections into the global information flow of the Web in ways that people expect to discover them. We need to move the description of the special, the unique, and the important to an industrial-scale level of efficiency, one that is tailored for machines as much as it is for end users. This is the only way that we are going to be able to achieve large-scale aggregation and relevance.

We need to consider together how to accomplish this goal. We need to have the courage to embrace a different approach, and with it establish a different kind of forum, a new type of venue, where we can do this collective thinking. We need a space in which we can collectively establish new practices and decide how to reduce the cost of what we do so that network-scale discoverability will be achievable and affordable. Only then will we be in a position to raise or break through the digital curtain.

The new Programs and Research group that is being created at OCLC as a consequence of the RLG-OCLC combination is intended to provide such a space and to become a venue for the collective thinking that will enable our current descriptive practices to change. The title of the August 2006 RLG Members Forum expresses our imperative: "More, Better, Faster, Cheaper." I do not know how to accomplish this goal, but I submit that it must be achieved and that it can only be achieved by our working together. Programs and Research will aim to provide the consensus and community building. I hope that in cooperation with other organizations, we will be able to link the outcomes to changes in practice that create a renewed and growing Web presence for cultural institutions of all types and sizes. This is a grand view of our mission and our importance as cultural institutions, but I believe it is an accurate one. I find it an exciting project, and one worthy of our collective efforts. 